\title{
Optimal Endogenous Neural Noise Speeds Reaction Time*
}

\author{
Lawrence M. Ward ${ }^{1,2}$, Aaron Kirschner ${ }^{3}$, Lauren Emberson ${ }^{1}$, and Keiichi Kitajo ${ }^{4}$ \\ ${ }^{1}$ Department of Psychology \\ ${ }^{2}$ Djavad Mowafagian Centre for Brain Health, University of British Columbia \\ ${ }^{3}$ Department of Medicine, McMaster University \\ ${ }^{4}$ RIKEN Brain Science Institute
}

Running head: Endogenous neural noise and reaction time

Corresponding author:

Lawrence M. Ward

Department of Psychology

2136 West Mall

University of British Columbia

Vancouver, BC V6T1Z4, Canada

1ward@psych.ubc.ca

Funded by: Natural Sciences and Engineering Research Council of Canada and Toyota Corporation

Conflicts of Interest: None declared

*A version of this work was presented at Fechner Day 2011 in Raanana, Israel 


\begin{abstract}
Objective: To determine whether the detection of weak visual stimuli is facilitated by a particular level of endogenous neural noise.

Method: We measured the EEG while subjects responded to the occurrence of weak luminance increments. We used independent component (IC) analysis and dipole fitting to locate the neural sources of EEG responses to the stimuli in occipital, frontal, and temporal cortex. We then determined the relationship between the variability of the frequency-specific oscillations of the relevant neural sources in the $1 \mathrm{sec}$ before stimulus presentation and the RT on hit (correct detection of the increment) trials.

Results: An intermediate amount of power variability yielded the fastest RTs. This nonlinear relationship was found for occipital and frontal cortical sources and was strongest for theta $(4-8 \mathrm{~Hz})$ and alpha $(9-14 \mathrm{~Hz})$ frequencies, but was also present for gamma $(30-50 \mathrm{~Hz})$ in frontal cortex.

Conclusion: These results support the idea that an intermediate level of endogenous neural noise optimizes the brain's response to a weak visual stimulus.
\end{abstract}

Keywords: endogenous neural noise, reaction time, visual detection, theta, alpha, gamma 


\section{Introduction}

It has been well documented that adding sensory noise to weak signals results in enhanced signal detection, a phenomenon termed "stochastic resonance" [1]. Recently we showed that the addition of exogenous noise to a sensory signal increases neural synchronization in response to weak auditory signals, both within and across brain regions responsive to the signal [2]. Importantly, models that assume that a certain level of endogenous noise is also involved in such detection both fit the data better [3], and also predict more stochastic resonance the lower is the endogenous noise [4]. It also has been proposed that the human brain can adjust its level of endogenous, or self-generated, neural noise in order to optimize neural processing [5]. A few previous studies have indicated that optimum levels of endogenous noise can facilitate responses to sensory stimuli (e.g., [6-7]), although it is not clear that what they observed was actually stochastic resonance (see [5] for the argument).

In a previous study of endogenous noise we showed that reaction time in visual detection is non-linearly related to pre-stimulus random-phase gamma power in a way that suggested stochastic resonance based on endogenous neural noise [5]. We were, however, unable to localize these effects in the brain, being limited to effects in scalp recordings. Also, we were still unsure of whether we had measured endogenous neural noise in the EEG or whether what we had seen was something else. Thus, in the present paper, we report an improved analysis of those same data. We used Independent Component Analysis (ICA) to separate the mixed volumeconducted signals from neural sources recorded by the EEG electrodes into independent components involved in the visual detection task that could then be localized to specific brain regions. ICA is an empirically- driven statistical approach to blind source separation, and does not rely on any assumptions about a brain model, which are only used in the localization. It thus facilitates discovery of the brain areas functionally involved in an experimental task [8]. We also explored several different ways of assessing the amount of neural noise in the $1 \mathrm{~s}$ period preceding stimulus onset, including sample entropy, and mean and variance of power in various frequency bands of the broad band time series of activation of the relevant ICs. In particular, we expected that power variance, which could be thought to reflect noisy synchronization of neurons within a brain region, would be nonlinearly related to RT and possibly also to oscillatory power generated by the stimulus.

\section{Methods}

\section{Subjects and EEG Recordings}

Six subjects (18-28 years, mean age 21, all male and right-handed) participated for pay. The study procedures were approved by the UBC Behavioural Research Ethics Board and all subjects gave written consent after having the procedures explained to them. EEG signals were recorded through 59 channels at standard 10-10 locations plus 3 channels at nonstandard nearinion sites and one channel for the bipolar electro-oculogram (EOG, electrodes $1 \mathrm{~cm}$ from outer canthi of both eyes). Electrode impedances were kept below $10 \mathrm{k} \Omega$ (amplifier input impedance $>$ $2 \mathrm{G} \Omega$ ). EEG and EOG were amplified with a gain of 20,000, bandpass filtered $(0.1 \mathrm{~Hz}-200 \mathrm{~Hz})$, digitized at $500 \mathrm{~Hz}$ and stored on disk for offline analysis. Scalp voltages were referenced to the right mastoid during recording and were re-referenced to the average reference during data analysis.

\section{Experimental Paradigm}


Subjects indicated detection of an occasional small luminance increment of a filled-in square, which was presented continuously on a black background on a computer monitor, by pressing the left arrow key on a keyboard with their right hand. Subjects responded as quickly and accurately as possible. Increment level for each subject was determined by adaptive staircase testing for the 50\% increment threshold and then adding one grey level of luminance. Before the adaptive staircase, subjects were presented with a small number of practice trials. Luminance increment duration was $100 \mathrm{~ms}$ and subjects were given $1000 \mathrm{~ms}$ after increment onset to respond. In order to prevent anticipation errors, time between trials varied randomly from 2000 ms to $3500 \mathrm{~ms}$, and a random $70 \%$ of the trials were stimulus present trials and $30 \%$ were stimulus-absent trials, during which no luminance increment occurred. Subjects ran twenty 50trial blocks, separated by self-timed breaks, resulting in 700 stimulus-present trials and 300 stimulus-absent trials per subject.

\section{Data Analysis}

The EEG data were analyzed with EEGLAB software [9], an open source MATLAB (Mathworks, Natick, USA) toolbox available at http://sccn.ucsd.edu/eeglab. EEG data contaminated by sources of artifacts (blinks, eye movements, muscle tension artifacts, and infrequent single-channel noise) were rejected using ICA. Decomposition of the continuous EEG into ICs was done separately for each participant. We used the EEGLAB runica algorithm, which is based on the infomax neural network algorithm [10], which exploits temporal independence to perform blind source separation. A scalp map for each IC was computed from the inverse of the IC weight matrix, giving the relative magnitude and polarity of the IC contribution at each electrode. This scalp map was then compared with the forward solutions for various single equivalent dipoles. The digitized 3-D locations of the electrodes on the scalp were first coregistered with the Montreal Neurological Institute (MNI) average brain. Single IC sources were then localized with the Dipfit2 algorithm in EEGLAB using a standard boundary element model. Only non-artifactual ICs with scalp maps having an inverse solution for a single dipole within Talairach (MRI) space (within the brain) of less than $15 \%$ residual variance were included in the subsequent analyses. Cluster analysis of all valid ICs, based on their dipole locations, was used to discover ICs that were common across subjects. Three relatively tight clusters of ICs contained at least one IC from each subject and each also showed an increase in power, relative to the pre-stimulus baseline, in theta and gamma frequency bands, and a decrease in alpha power, shortly after stimulus onset, indicating visual stimulus processing (see Results and Discussion). Three subjects had more than one IC in these clusters and in these cases the IC nearest the centroid of the cluster was selected. The resulting three clusters of six ICs were analysed further. Other ICs also showed stochastic resonance-like effects, implicating other brain areas, but these are not reported here because they were not general to all of the subjects.

Wavelet analysis was used to measure phase and amplitude of oscillations at frequencies from $3 \mathrm{~Hz}$ to $70 \mathrm{~Hz}$ for each epoch from $-1000 \mathrm{~ms}$ to $+1000 \mathrm{~ms}$ re stimulus onset for hits only. The numbers of hits per subject were $464,344,502,212,419$, and 225 , mean $=361$, or an average of $51.57 \%$ hits. RTs for hits were sorted into six subsets for each subject based on variance of oscillatory amplitude (spectral power) in each of three frequency bands: theta (4-8 $\mathrm{Hz}$ ), alpha (9-14 Hz), and gamma (30-50 Hz) (lowest to highest 1/6 of total number of epochs available). Mean RTs for each subset were normalized relative to the maximum for each subject separately and the normalized values were subjected to ANOVAs and curve fitting. Average spectral power in the post-stimulus-onset period from 200-600 ms (theta), 300-600 ms (alpha), 
and 400-600 ms (gamma) was also computed for each subset of trials and a similar analysis applied. We also computed sample entropy of the broadband IC activations and used these as the basis for the same type of analysis as described above.

\section{Results and Discussion}

Figure 1 displays the brain locations of left occipital lobe (centroid at Talairach $-22,-77$, 11; Brodmann 17) and medial frontal (centroid at Talairach -9, 43, 24, Brodmann 9) ICs. The cluster in temporal cortex revealed no changes in RT as a function of power or power variability and so is not shown. Figure 2 shows the average spectral power for occipital and frontal sources as a function of time and frequency. As mentioned earlier, increases in relative power in theta and gamma bands accompanied by a decrease in alpha power indicates active processing of the stimulus in these brain regions.

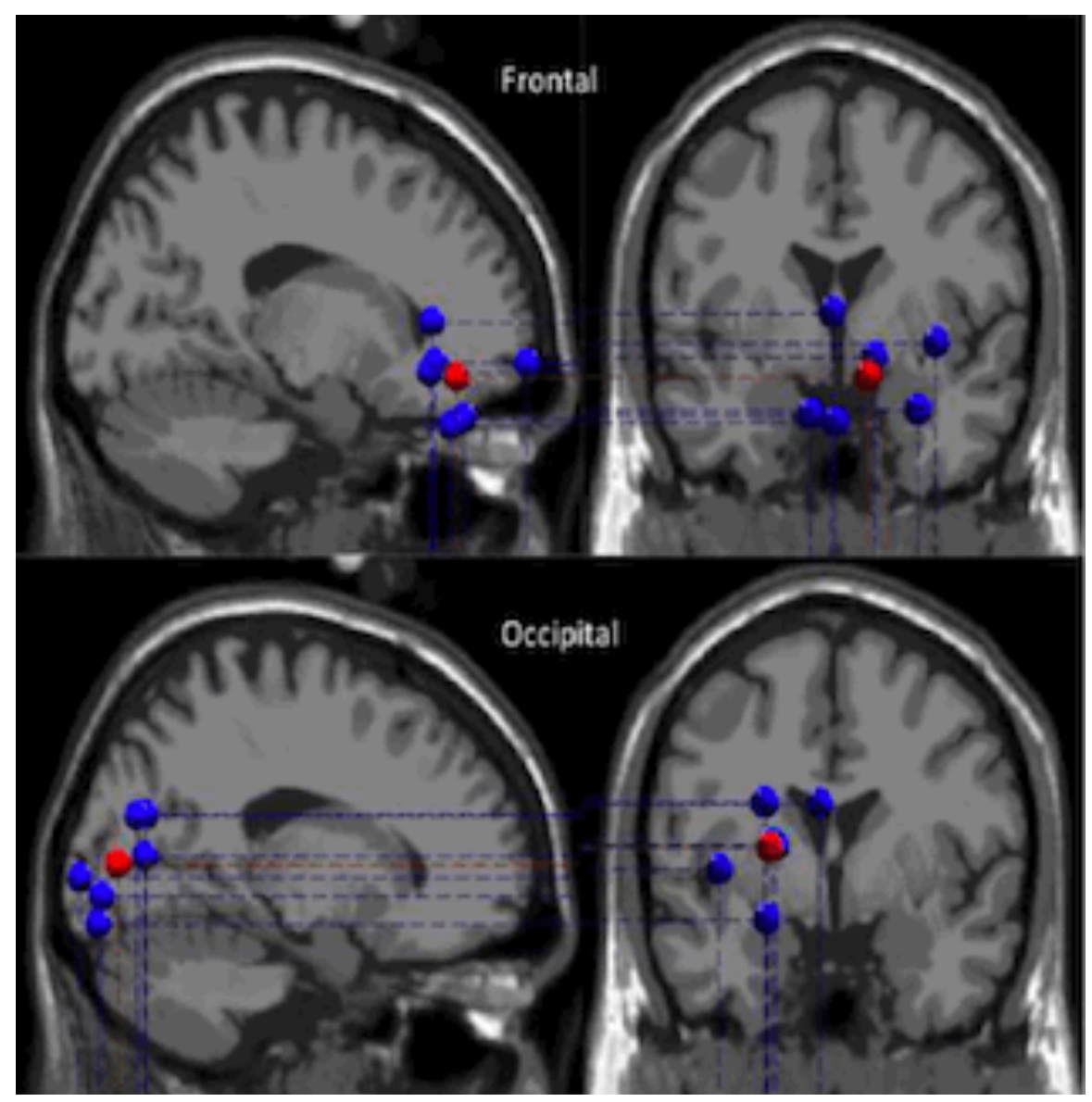

Figure 1. Dipole locations of ICs selected for power analysis. Blue spheres indicate individual subjects' ICs; the red sphere is the centroid. 

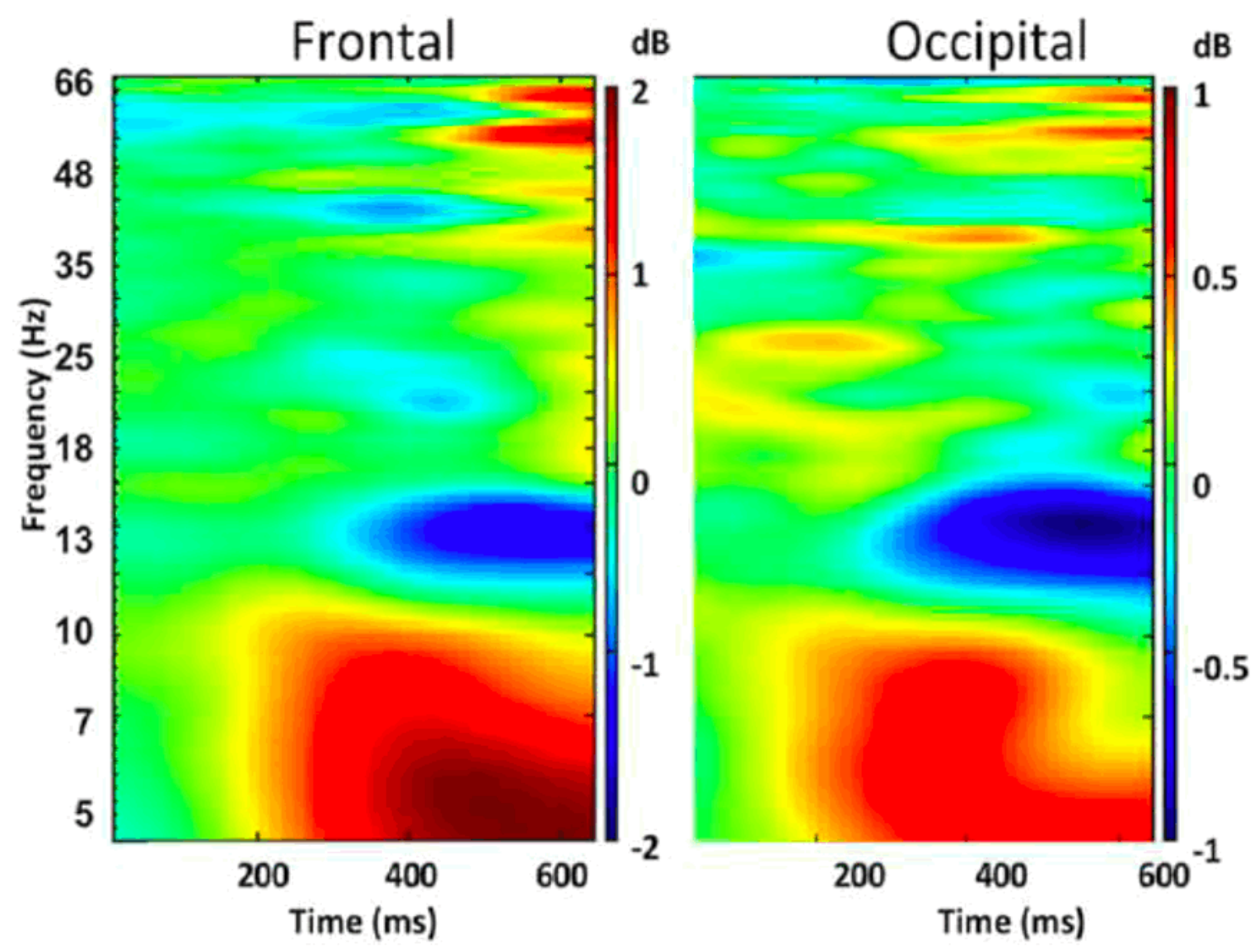

Figure 2. Time-frequency plot of average relative power after stimulus onset on hit trials.

We found no relationship between sample entropy, which measures complexity, and RT, and also none between RT and average power in any frequency band, and so these results are not reported. There were strong linear relationships between pre-stimulus power variance and poststimulus spectral power for same-frequency analyses, and more complex relationships for different pre- and post-stimulus frequencies. These did not seem to add anything to the present story, however, and so are not reported further.

Figure 3 (occipital) and Figure 4 (frontal) display graphs of RT as a function of prestimulus power variance in theta, alpha, and gamma (frontal only) frequency bands. In occipital cortex the RT differences averaged $35 \mathrm{~ms}$ (theta) and $22 \mathrm{~ms}$ (alpha) from the lowest powervariability subset of trials to the subset with the lowest RT, and the differences over the 6 subsets are significant for both frequencies (theta: $F=3.02, d f=5,30, p<0.03$; alpha: $F=4.71, d f=$ $5,30, p<0.003$ ). Notice that the quadratic functions fit nicely, with high (alpha: 0.90) and moderate (theta: 0.62) $r^{2}$ values, whereas the linear functions do not fit at all, having $r^{2}$ values of virtually zero. In frontal cortex the RT differences averaged $16 \mathrm{~ms}$ (theta), $17 \mathrm{~ms}$ (alpha), and 23 $\mathrm{ms}$ (gamma) as above, and they are significant for theta $(F=3.52, d f=5,30, p=0.01)$, and alpha $(F=6.52, d f=5,30, p=0.0003)$ but not for gamma $(F=1.91, d f=5,30, p=0.12)$, although the quadratic fit $\mathrm{r}^{2}$ for theta $(0.89)$ and gamma $(0.87)$ were both quite large, with alpha 
(0.64) somewhat lower but still significant. Again, linear functions fit very poorly at any frequency.
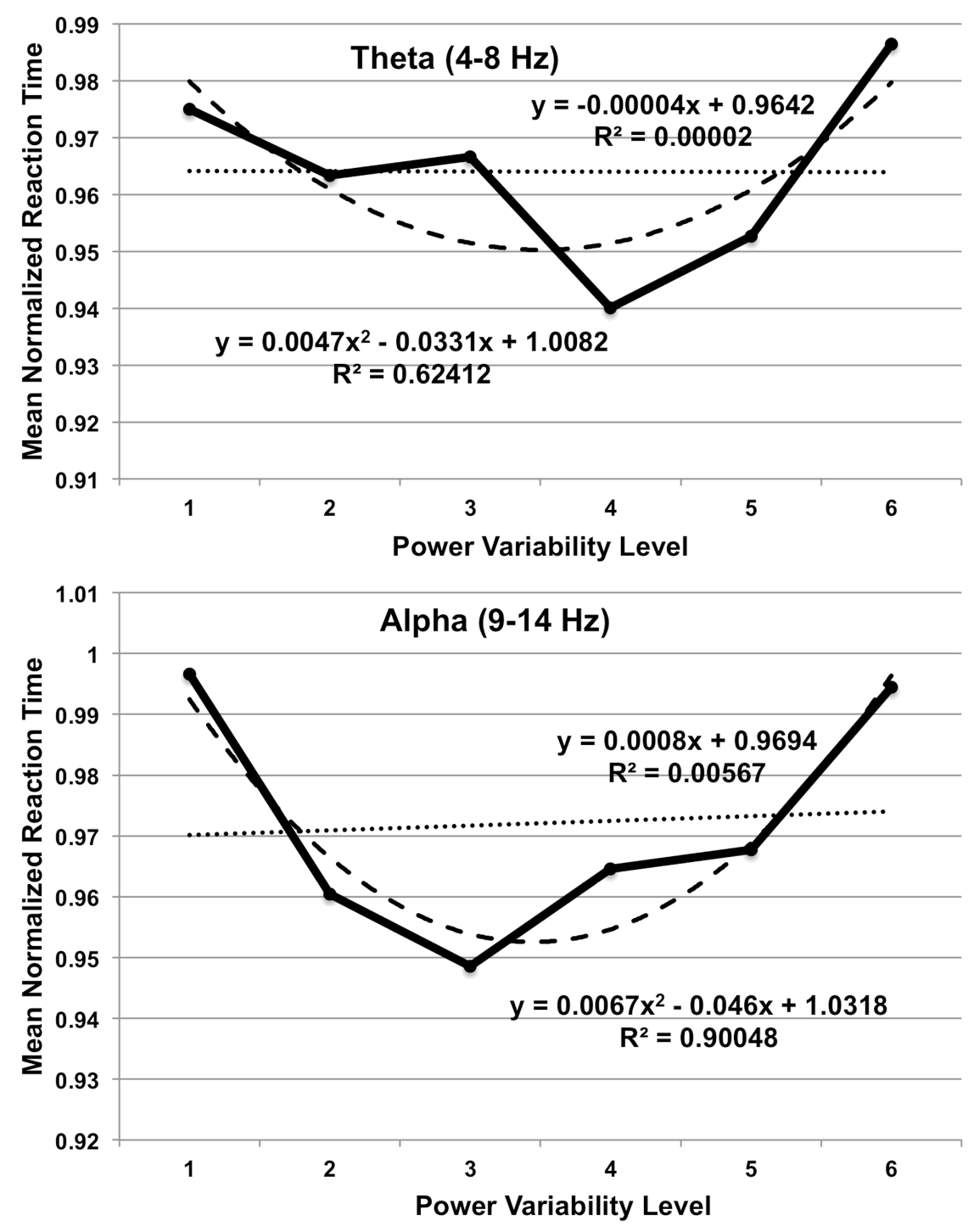

Figure 3. Mean normalized reaction time as a function of theta or alpha power variability in occipital cortex. Dark lines are data, dotted lines are linear trends with equations above, and dashed lines are quadratic trends with equations below. 


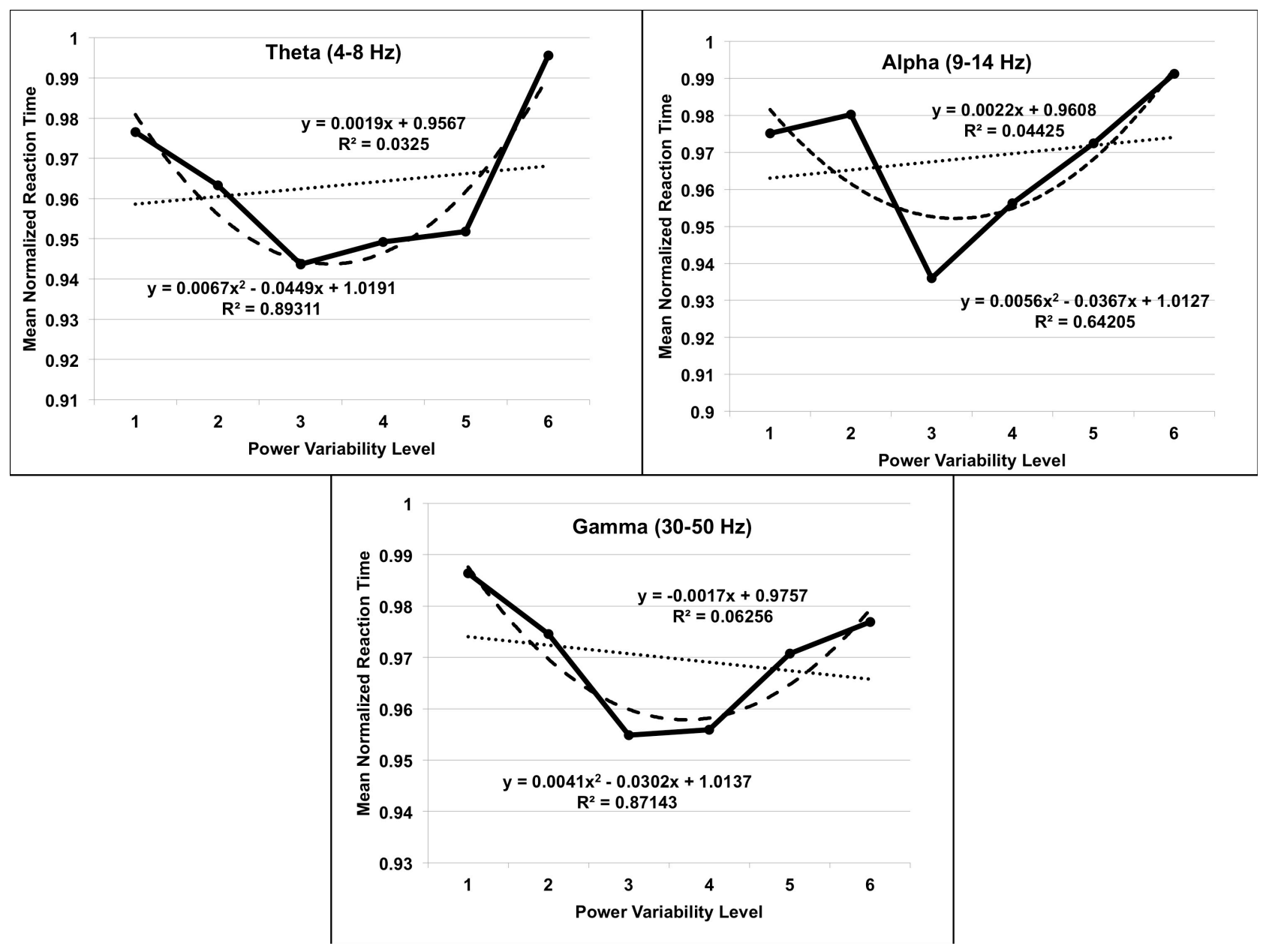

Figure 4. Mean normalized reaction time as a function of theta, alpha, and gamma power variability in frontal cortex. Dark lines are data, dotted lines are linear trends with equations above, and dashed lines are quadratic trends with equations below.

EEG electrodes only record potentials from groups of neurons that either (1) have a population dynamic oscillating synchronously in a given frequency range [11], or (2) act as filter or resonator for noise in some frequency band [12]. Thus, power variance can be interpreted either as the magnitude of noisy synchronization or as the efficiency of a noisy filter or resonance. Our data indicate that RT is faster when power variance in theta and alpha, and possibly gamma, frequency bands is in an intermediate range. We take this to indicate that an intermediate amount of endogenous noise generates the optimum neural signal involved in responding to this very weak stimulus. This indicates that the brain response to a stimulus is sensitive to the magnitude of endogenous neural noise. Because synchronization of real and model neurons is modulated by noise [13], and optimized by an optimum noise level, our data are consistent with the idea that a synchronous population dynamic optimizes response to a stimulus.

In a recent paper McDonnell and Ward [14] termed findings such as these "stochastic facilitation," because they do not fit with the standard paradigm of stochastic resonance, in which external noise is added to weak signals in order to improve detection of those signals. In their 
suggested paradigm, once such a finding is reported the next steps are (1) to model the system in a biologically realistic way, and (2) to determine if the noise is really critical to the relevant computational goal of the system by subtracting it out of both model and real systems. These steps, as well as further analyses to determine whether pre-stimulus variability influences other aspects of the brain response to the stimulus, such as oscillatory synchronization and information transfer, or other behavioral aspects, such as attention focus (cf. [15]), must now be carried out.

\section{References}

1. Moss, F., Ward, L.M. \& Sannita, W.G. (2004). Stochastic resonance and sensory information processing: A tutorial and review of applications. Clinical Neurophysiology, 115, 267281.

2. Ward, L.M., MacLean, S.E., Kirschner, A. (2010). Stochastic resonance modulates neural synchronization within and between cortical sources. PLOS ONE, 5(12): e14371.

3. Ward, L.M. (2004). Psychophysics of stochastic resonance. Fluctuations and Noise Letters, 4, L11-L21.

4. Aihara, T., Kitajo, K., Nozaki, D. \& Yamamoto, Y. (2008). Internal noise determines external stochastic resonance in visual perception. Vision Research, 48, 1569-1573.

5. Emberson, L., Kitajo, K. \& Ward, L.M. (2007). Endogenous neural noise and stochastic resonance. In S. Bezrukov (Ed.), Noise and Fluctuations in Biological, Biophysical, and Biomedical Systems (pp. 6602OT1-6602OT12). Proceedings of SPIE Vol. 6602. Bellingham, WA: SPIE.

6. Winterer, G., Ziller, M., Dorn, H., Frick, K., Mulert, C., Dahhan, N., Herrmann, W.M. \& Coppola, R. (1999). Cortical activation, signal-to-noise and stochastic resonance during information processing in man. Clinical Neurophysiology, 110, 1193-1203.

7. Linkenkaer-Hansen, K., Nikulin, V., Palva, S., Ilmoniemi, R., Palva, J. (2004). Prestimulus oscillations enhance psychophysical performance in humans. Journal of Neuroscience, 24, 10186-10190.

8. Viola, F. C., Thorne, J., Edmonds, B., Schneider, T., Eichele, T., \& Debener, S. (2009). Semiautomatic identification of independent components representing EEG artifact. Clinical Neurophysiology , 120, 868-877.

9. Delorme, A., \& Makeig, S. (2004). EEGLAB: An open source toolbox for analysis of single trial EEG dynamics including independent component analysis. Journal of Neuroscience Methods , 134, 9-21.

10. Bell, A. J., \& Sejnowski, T. J. (1995). An information-maximization approach to blind separation and blind deconvolution. Neural Computation, 7, 1129-1159.

11. Nunez, P.L. (2000) Toward a quantitative description of large-scale neocortical dynamic function and EEG. Behavioral and Brain Sciences, 23, 371 - 437.

12. Burns, S.P., Xing, D. \& Shapley, R.M. (2011). Is gamma-band activity in the local field potential of V1 cortex a "clock" or filtered noise? The Journal of Neuroscience, 31, 9658 -9664 .

13. Ward, L.M. (2009). Physics of neural synchronization mediated by stochastic resonance. Contemporary Physics, 50, 563-574.

14. McDonnell, M.D. \& Ward, L.M. (2011). The benefits of noise in neural systems: bridging theory and experiment. Nature Reviews: Neuroscience, 12, 415-425. 
15. MacDonald, J.S.P., Mathan, S., \& Yeung, N. (2011). Trial-by-trial variations in subjective attentional state are reflected in ongoing prestimulus EEG alpha oscillations. Frontiers in Psychology, 12, doi: 10.3389/fpsyg.2011.00082. 